\title{
INFRAESTRUCTURAS PARA POROSIDADES SELECTIVAS El limboscape de Ceuta
}

Palabras clave

\section{Frontera}

Excepción

Legalidad

Europa

África
En una de las fronteras más complejas del

mundo, el límite físico se materializa en lo que parece ser una simple reja. Esto, sin embargo, no significa que el límite sea débil. Como indica este texto, la infraestructura fronteriza en Ceuta es un ensamblaje complejo de artefactos físicos, constructos legales y calculadas excepciones a estas reglas que, a fin de cuentas, generan un límite con porosidad selectiva.

euta sirve para amplificar y resaltar la contradictoria lógica del manejo de las fronteras europeas, porosas e impenetrables a la vez. Propongo examinar la condición de frontera como una infraestructura legal, económica, política y material y, en consecuencia, analizarla como una compleja realidad construida y no sólo como una línea divisoria. Así, la frontera se repiensa como algo más amplio, un ecosistema en sí mismo que genera y activa sus propias coreografías en el espacio.

Ceuta es la última frontera entre dos continentes: Europa y África. Además, se encuentra en el límite de dos masas de agua, el océano Atlántico y el mar Mediterráneo $y$, como tal, flanquea la principal puerta de entrada a este último. Finalmente, se ha consolidado a lo largo de la historia como la frontera cultural y religiosa entre el cristianismo y el islam. La especificidad de la geolocalización de Ceuta y la escala de la ciudad proporcionan una condición fronteriza muy paradójica, al punto que la ciudad completa podría entenderse como una frontera: es, en sí misma, una enorme cantidad de contradicciones legales y territoriales que transforman este territorio en un limboscape [paisaje-limbo] (Ferrer-Gallardo y Espiñeira, 20I5). En su historia, 

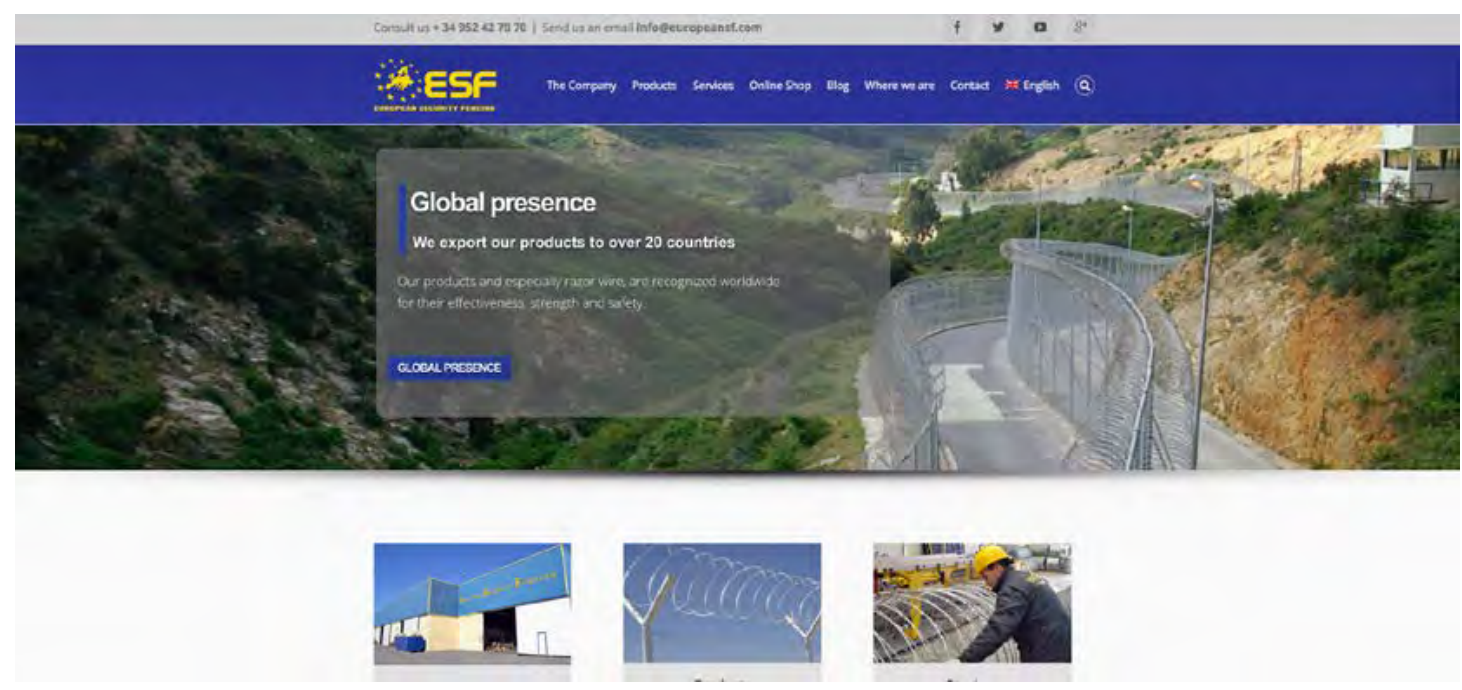

Ceuta ha acumulado una gran diversidad de encuentros

donde la soberanía política y cultural, al igual que las fronteras de la ciudad, han estado en disputa.

El propio origen de la definición de la frontera de European Security Fencing website. Ceuta - y el consiguiente reconocimiento de pertenencia al territorio que circunscribe - es controversial. Dado que el sedentarismo es la condición regular para pertenecer a un lugar, todas las soberanías nómades que han pasado por este territorio quedan automáticamente eliminadas. Tomar esta condición como punto de partida ya implica una caracterización compleja y en disputa con la pertenencia a este territorio.

España se incorporó a la Unión Europea (UE) en I986. En I991, y como parte de esta nueva integración, adhirió al Acuerdo de Schengen, un pacto territorial que definía el 'espacio Schengen' como una zona sin fronteras que permitiría la libre circulación de ciudadanos de los países involucrados, sin necesidad de una visa. Como consecuencia inmediata de este acuerdo, Ceuta se convirtió en la nueva frontera sur de la UE (la única frontera terrestre, junto a la ciudad de Melilla, de la U E con África). Como parte de España, Ceuta pertenece en términos políticos a la UE, pero, contradictoriamente, su territorio se encuentra fuera del espacio Schengen. De esta forma, Ceuta se convirtió en una frontera europea fuera del continente europeo; una frontera extraterritorial.

Pero las cosas son aún más complejas. Ceuta está inscrita dentro de una doble frontera. Hacia el sur, el límite con Marruecos se construye mediante una reja y, hacia el norte, la frontera es el mar Mediterráneo. En este último, el terminal portuario del ferry que conecta por agua con la península ibérica construye, legalmente, las puertas de la frontera. Dicho terminal flanquea uno de los lados del estrecho de Gibraltar, el único acceso al Mediterráneo desde el océano Atlántico. El otro flanco es el equivalente simé- 


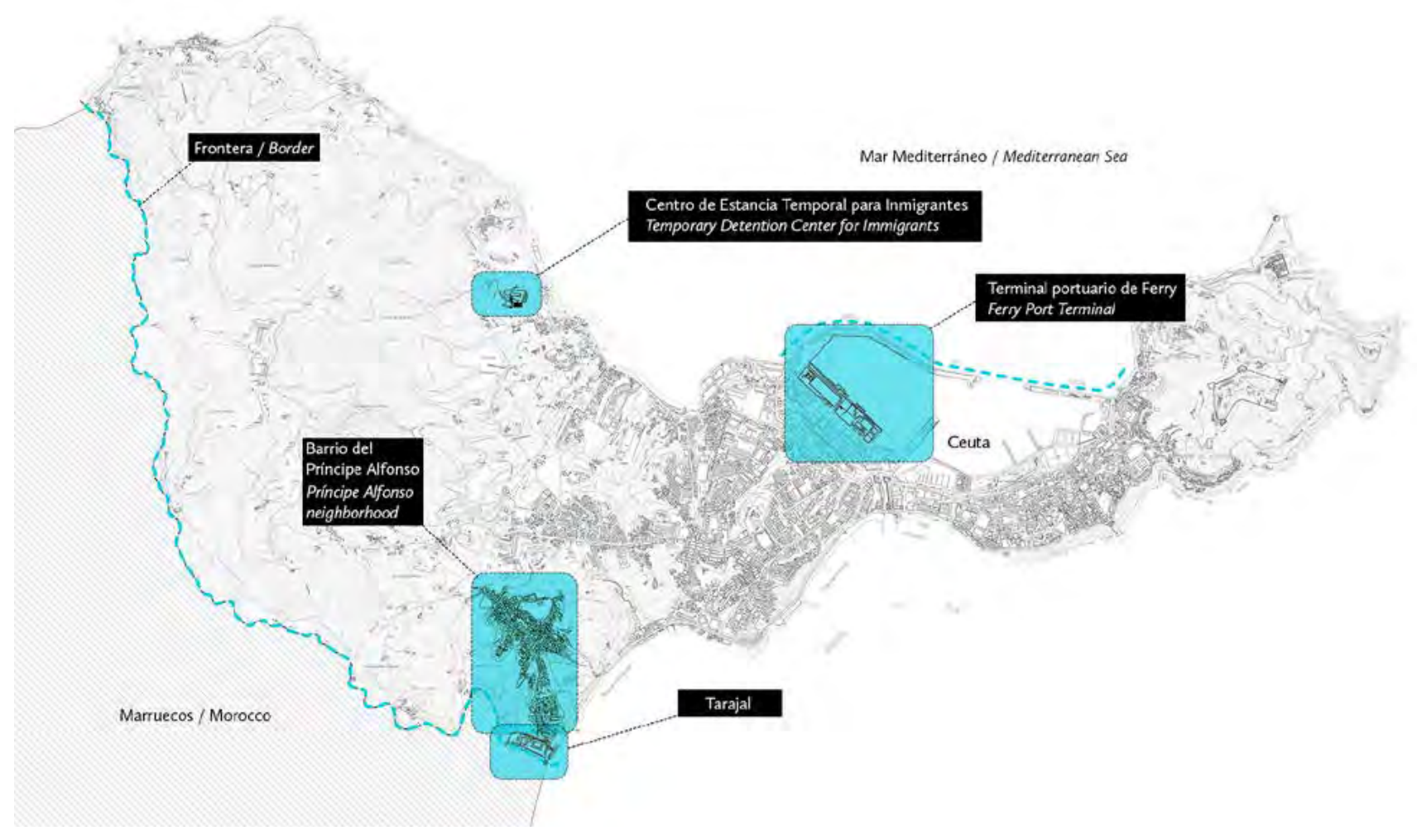

Mapa de Ceuta, España. Map of Ceuta, Spain. trico de Ceuta ubicado en la península ibérica, el Peñón de Gibraltar, transferido al Reino Unido en I7ız en un artículo del Tratado de Utrecht. Desde entonces, y pese a los innumerables reclamos del territorio por parte del Gobierno Español y con mayor énfasis luego de los acuerdos de descolonización dirigidos por la $\mathrm{ONU}$, ha operado como un punto militar estratégico para el control de las rutas de navegación del mar Mediterráneo. La disputa sobre este pequeño territorio no es casual, ya que gracias a dicha porción de suelo extraterritorial el Reino Unido tiene su propio acceso al Mediterráneo. Históricamente, el control militar ha sido la función principal de Ceuta y Gibraltar, y ambas pueden leerse como resultados tangibles de acuerdos políticos y un proceso de reforzamiento de la seguridad.

Aquí nos centraremos en las infraestructuras que materializan los aparatos jurídicos en juego: por una parte, describiendo el papel de Ceuta en el proceso de 'Schengenización' y específicamente el rol que desempeña la reja; y por otra, revelando los dos resultados de esta compleja relación. En primer lugar, la porosidad selectiva que revelan las regulaciones fronterizas en Ceuta (el reflejo y la amplificación del comportamiento fronterizo contradictorio y paradójico de la UE); y en segundo lugar, un análisis de las arquitecturas que generan esta porosidad selectiva y la realidad cotidiana de sus resultados.

Infraestructuras legales y físicas:

el Acuerdo de Schengen

El rol de Ceuta en los planes de reforzamiento de la seguridad en la Unión Europea va más allá de lo que se puede ver a simple vista. A través de su propia materialización, la reja que divide el territorio europeo/español del marroquí desempeña un papel muy complejo. Los dichos de Etienne 
Balibar, que se refieren a las estrategias europeas como «el nuevo apartheid», refuerzan los alegatos sobre «la construcción europea como una institución 'postnacional' (...) dedicada a que ningún ámbito de vida social quede libre de ser afectado por sus requisitos de seguridad» (Balibar, 20Ir:I). Entre las construcciones realizadas en nombre de la seguridad', la reja de Ceuta representa la materialización in situ de dichas fronteras políticas; un límite construido a través de la historia. Como también plantea Balibar (20I1:4-5), «los muros no son estructuras pasivas», sino «líneas de cristalización», articulaciones activas y orgánicas que reflejan explícitamente las decisiones políticas. Por ejemplo, los cambios en la altura de la reja pueden inscribirse dentro de períodos políticos muy precisos y son un reflejo de las decisiones del Gobierno Español contra las diversas oleadas de migrantes. Además, considerando la materialización física de la reja, su disposición representa la dualidad que encarna el límite: en este caso, una reja doble con un espacio de aproximadamente 4 metros entre ellas, que es vigilado por patrullas a pie. En el lado correspondiente al territorio español, la reja está rodeada por una línea de postes de luz muy cercanos entre sí, seguida por una carretera de dos pistas para el control de automóviles patrulleros. La propia existencia de dos rejas representa los dos poderes que vigilan la frontera: el marroquí y el español. Esta dualidad es llevada al extremo al considerar que los inmigrantes que mueren en la sección marroquí de la reja son considerados un problema de los marroquíes, mientras que los cuerpos que fracasan en el segundo salto - es decir, la segunda reja correspondiente a la vigilancia española - son responsabilidad de los españoles. Así, lo que ocurre en el medio generalmente se convierte en un tira y afloja entre ambos países. Es la propia materialidad de la separación la que articula físicamente la dualidad de la legalidad.

Entre las múltiples y complejas ambivalencias del caso está el rol de la reja de Ceuta como ejemplo de excelencia en el diseño de seguridad. Como en todas las prácticas de construcción, la normalización - y la naturalización - de esta práctica estandarizada que sirve a las agendas políticas actuales, se realiza a través de un certificado de calidad. En este caso, vemos que la propia reja de Ceuta es el sello de calidad para el diseño de seguridad fronteriza. Entre las empresas que materializan este complejo ecosistema de fronteras construidas en Europa se encuentra European Security Fencing (ESF), una iniciativa privada fundada en Málaga en I975 y único fabricante de rejas de alambre en el continente, con sede ampliada en Berlín. Las estrategias fronterizas de Europa, junto a centros penitenciarios y plantas nucleares en cinco continentes, son los principales clientes de la compañía. Como empresa que ofrece un servicio - diseño de seguridad, en este caso - las rejas de Ceuta y Melilla son las obras maestras utilizadas para ejemplificar la calidad de su marca. Así, la reja de Ceuta se convierte en referencia y garantía del diseño de seguridad europeo, algo claramente declarado en el slogan de E S F : «instalando rejas de alambre en Ceuta y Melilla desde i989».

Aunque la reja es la primera materialización de las políticas fronterizas, así como la más visible, la articulación del territorio de Ceuta va más allá de la reja. Es un 
Reja fronteriza de Ceuta. / Ceuta border fence. Mario Sánchez Bueno Creative Commons territorio donde varias capas legales se superponen sin coincidir, dejando vacíos legales que posibilitan realidades incongruentes. Es también un territorio donde diversos dispositivos arquitectónicos participan en la materialización de las regulaciones de migración.

Dos cruces de caminos:

los inmigrantes subsaharianos

Los inmigrantes subsaharianos que logran entrar en Ceuta (ya sea saltando la reja o nadando) llegan a un territorio que está suspendido entre la frontera con Marruecos, terrestre y enrejada, y la frontera del mar Mediterráneo, acuática y controlada. Una vez dentro, los migrantes permanecen suspendidos en este limboscape legal en el que sus «trayectorias hacia 'Europa-U E' se suspenden espacial y temporalmente» (Ferrer-Gallardo y Espiñeira, 2015:25I). La paradoja aparece cuando esta inmovilidad encapsula cuerpos en un sitio donde no pueden quedarse ni irse. Es la materialización misma de la contradicción, ya que son forzados a permanecer en un lugar donde tienen prohibido quedarse, un territorio cerrado donde la incapacidad de absorber esta ilegalidad surge como una de las principales características urbanas de la ciudad.

Aunque la única solución legal concebida es la repatriación, el estatus de estos inmigrantes se caracteriza por su imposibilidad de ser deportados, principalmente porque su país de origen no los reclama (ya que eso supondría asumir los costos de la deportación). En otras palabras, al insistir en su derecho soberano de expulsión, el Estado provee un marco que contempla la posibilidad de expulsarlos del país; sin embargo, no existe un marco que les permita quedarse en Ceuta. Dado que esta última opción ni siquiera se considera como probabilidad, no hay un reconocimiento legal de su posibilidad y, por ende, no existe la necesidad de solución alguna.

Como la única posibilidad reconocida es que estos inmigrantes permanezcan en Ceuta hasta su deportación, la única infraestructura provista es el Centro de Estancia 
Temporal para Inmigrantes (CET1), un edificio capaz de albergar hasta 500 inmigrantes. Con forma de prisión enrejada y un patio central, este edificio es la única infraestructura existente para alojar a la población inmigrante. Sin embargo, se estima que alrededor de 2.000 inmigrantes permanecen en Ceuta por más de 5 años; de ellos, alrededor de I.40o se concentran en el CET I mientras que el resto, un total incierto, viven repartidos por el bosque o deambulan por la ciudad sin poder instalarse en ninguna casa o zona residencial que no sea la calle. Aunque las cifras pueden parecer menores, su impacto es extremadamente inquietante. Una lectura proporcional de estas cifras permite entender que casi un $2 \%$ de los habitantes de Ceuta vive en el CET I, Con una superficie total de $4.956 \mathrm{~m}^{2}$, este edificio tiene una densidad de 0.28 habitantes por $\mathrm{m}^{2}$ (280.0oo habitantes por $\mathrm{km}^{2}$ ), mientras que la densidad promedio de Ceuta es de 4.Ioo habitantes por $\mathrm{km}^{2}$. Esta disparidad también se refleja las contradictorias prioridades de inversión: se destinaron alrededor de 289 millones de euros en el control fronterizo, pero sólo se gastaron 9 millones para proporcionar atención primaria e infraestructura para albergar a los inmigrantes.

$\mathrm{El}$ «refugiado sin tierra» de Arendt o el «estatus de refugiado» de Agamben son paradigmas que representan el estatus constitutivo de una estructura recientemente articulada y distinta al Estado-nación (Agamben, 2000:I425). Sin embargo, al considerar a los migrantes detenidos en Ceuta aparece una cualidad distinta: el propio acto de reconocimiento. Como Ceuta no es un campo de refugiados sino una ciudad, no se les considera refugiados. Es decir, cuando no se reconoce el limbo en el que se encuentran, aparece la complejidad de enfrentar discursivamente el estatus de estos inmigrantes. De hecho, el funcionamiento de la legalidad en Ceuta comparte muchas de las características indicadas por Agamben en su definición del campamento:

En él, el estado de excepción, que era esencialmente una suspensión temporal del Estado de derecho [en el caso de Ceuta sólo para algunos pero para otros no], adquiere una disposición espacial permanente que, como tal, permanece constantemente fuera del estado normal de ley. (...) El campamento es un pedazo de territorio fuera del orden jurídico normal (Agamben, 2000:39-40).

La paradoja surge cuando ambos órdenes jurídicos cohabitan. De esta forma, los ciudadanos de Ceuta ocupan legalmente el mismo territorio sobre el que 'otras personas', circulan bajo un régimen de excepción que no les permite abandonar un espacio delimitado por esta jurisdicción excepcional. Ceuta es, entonces, una ciudad para algunos y un campo de refugiados para otros.

Cuando un inmigrante entra en Ceuta cree que está llegando a un estado de libertad en la tierra prometida europea; sin embargo, una vez allí, su escape se convierte en una trampa sin salida.

Dos cruces de caminos:

las porteadoras marroquíes

En contraste con el estatus de los migrantes subsaharianos, que persiste con un resultado poco claro y no 
Reja fronteriza de Ceuta. Ceuta border fence. Mario Sánchez Bueno Creative Commons planificado, el estatus de los marroquíes es muy explícito. De hecho, la condición para la circulación de marroquíes en Ceuta es una de las pocas excepciones al Acuerdo de Schengen. Menos diseñado ha sido, sin embargo, el límite al que se puede llevar esta excepción.

Antes del Acuerdo de Schengen, y según la Ley de Extranjería de 1985, la mayoría de los ciudadanos musulmanes establecidos en Ceuta y Melilla no tenían acceso a la ciudadanía española. Luego de intensas protestas de los habitantes musulmanes en estas ciudades, se llevó a cabo una regularización masiva. Sin embargo, mientras las antiguas fronteras simbólico/religiosas se estaban disolviendo dentro de estas ciudades, nuevas líneas divisorias comenzaron a aparecer entre las comunidades fronterizas de ambos lados.

En I99I las fronteras euroafricanas (Ceuta y Melilla) se convirtieron en los fronteras exteriores del área Schengen. El mismo año se televisaron las primeras muertes de migrantes que intentaban cruzar ilegalmente el estrecho de Gibraltar. También en I99I se implementó una excepción cuidadosamente planeada del acuerdo, que permitió un flujo selectivo de ciertos ciudadanos marroquíes: la frontera de Schengen era hermética para la mayoría de ellos, pero era muy porosa para los ciudadanos del territorio de Tetuán. Dicha porosidad se concretizó en una visa de 24 horas que generó un tránsito constante de 30.000 a 40.000 personas diarias (Ferrer Gallardo, 2008:136).

Además de esta legalidad de Schengen, también existe un marco económico y fiscal muy complejo entre Ceuta/ España y Marruecos. Por un lado, Ceuta tiene un marco fiscal excepcional que, dada la gran diferencia económica entre ambos lados de la frontera, convierte a Ceuta en la arena perfecta para el intercambio informal. Pero esta no es

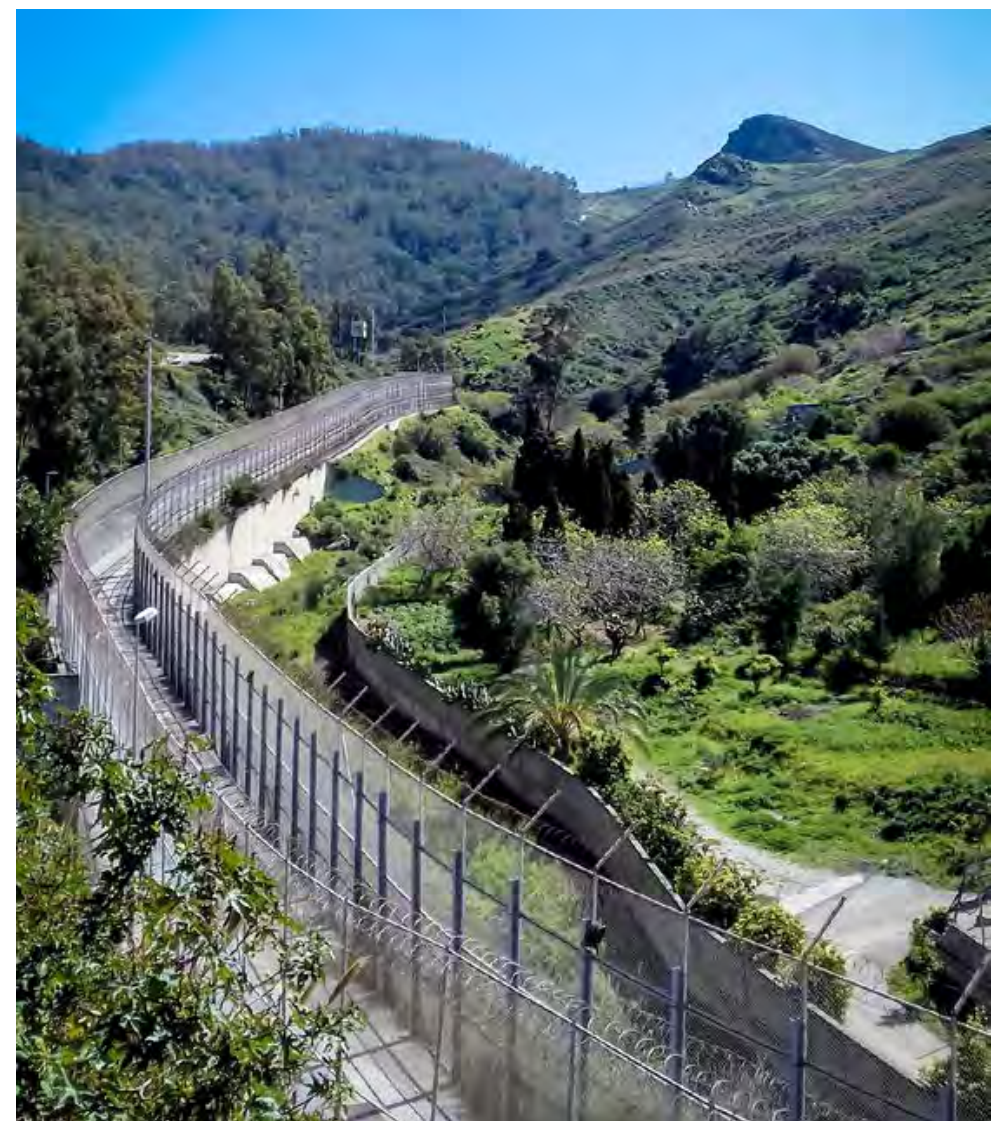


la única condición excepcional respecto al intercambio económico y comercial. Por sobre este conflicto está el extraño hecho de que no hay una frontera aduanera reconocida entre Marruecos y España. Aunque la razón oficial de esto parece ser política - Marruecos argumenta que reconocer una aduana implicaría reconocer la soberanía española sobre ese territorio - la economía informal que genera esta condición permite especular que la verdadera razón son los intereses económicos y comerciales. Sin fronteras aduaneras no hay una forma oficial de hacer circular las mercancías. Pero a su vez, los cuerpos humanos tienen todo el derecho a circular bajo el 'régimen del viajero', que permite a una persona y su equipaje cruzar libremente la frontera.

Con ambas regulaciones en juego - la fiscal, que permite la circulación de un cuerpo y un equipaje, y la excepción de Schengen que permite a una persona marroquí entrar en el territorio español/europeo durante 24 horas - el cuerpo humano de algunos marroquíes se convierte en el único vehículo para cruzar mercancías a través de la frontera. El resultado optimizado de este arreglo son las mujeres porteadoras que llevan hasta $70 \mathrm{~kg}$ embutidos en su propia ropa y en una bolsa gigante en su espalda.

El marco legal que posibilita esta economía informal está respaldado por dos dispositivos físicos: el cuerpo y los parques industriales que sirven para almacenar las mercancías traídas por estos cuerpos. Este espacio de almacenamiento es el polígono industrial El Tarajal, un complejo de almacenes cerca del Barrio del Príncipe, la zona musulmana de Ceuta.

Como la legalidad sólo permite que el viajero cruce caminando, la ubicación de este almacenamiento no puede estar lejos de la frontera. No es coincidencia que El Tarajal esté literalmente fijado al borde: su pared posterior es, de hecho, el muro fronterizo, siendo el único tramo en la que la reja doble se reemplaza por un muro de hormigón. Como contraparte material de la porosidad selectiva de la frontera, aquí no es visibilidad, sino opacidad, lo que se necesita para ocultar lo que sucede dentro de las paredes de El Tarajal, aun cuando es de conocimiento general.

Junto al El Tarajal, la frontera tiene su entrada principal: el tramo donde el muro se disuelve y es sustituido por un gran techo que aloja los pequeños quioscos de puntos de control fronterizo, donde se solicitan los pasaportes entre Marruecos y España. Esta es la puerta que usan las mujeres porteadoras para entrar a España, sin cargas. Sin embargo, no salen con su bulto por la misma puerta; más bien, dejan España para volver a entrar a Marruecos por la puerta trasera, que está un poco al oeste y es, literalmente, una apertura en el muro de hormigón. Tras cruzar esta puerta, las mujeres porteadoras ya están en Marruecos pero en medio del entorno natural. La cuidadosa articulación arquitectónica de la reja/ muro, a través de su puerta principal y posterior, es la coreografía de una confluencia paradójica de circulaciones previamente diseñada por medio de articulaciones legales. Así, la arquitectura de El Tarajal y la de ese tramo de la frontera son parte de un diagrama de relaciones políticas y de poder (Weizman, 2006:90) hacia ciertos cuerpos y hacia todo tipo de mercancías.

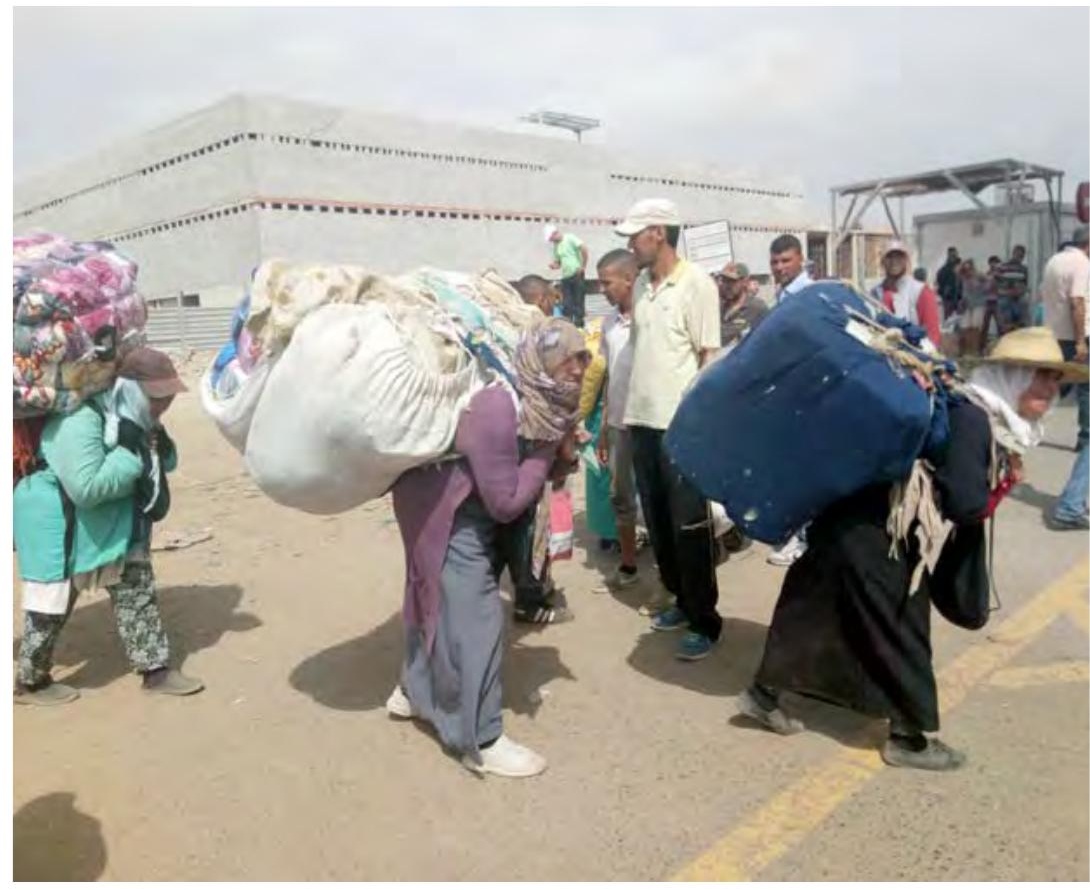

Caravana de porteadoras. Woman porters' caravan. Creative Commons 
Contrastando con el discurso liberal, «que insiste en la libre circulación como una especie de derecho natural» (Balibar, 20II:5), las múltiples materializaciones de las fronteras señalan realidades paradójicas. Considerando las articulaciones jurídicas que permite la realidad de las porteadoras, podemos preguntarnos: ¿qué es lo que puede circular libremente? ¿Son los ciudadanos de Tetuán? ¿Son las mercancías? ¿Es la fuerza laboral que encarnan esos inmigrantes marroquíes? Cualquiera sea la razón para producir una excepción al acuerdo de Schengen, lo cierto es que ha sido diseñada sin considerar los efectos sobre los sujetos involucrados en estas circulaciones.

La excepción de Schengen puede entenderse, según Balibar (20II:I8), como «inclusión y exclusión, legalidad e ilegalidad, admisión y represión», especialmente al considerar las demandas de Mezzadra sobre la funcionalidad de las fronteras, que justifica las controvertidas combinaciones. Entre estas contradicciones, las reglas de circulación del capital sobre una base globalizada hacen de esta funcionalidad la excusa perfecta para la circulación de la fuerza laboral (Balibar, 20Ir:I8). Es esta subversión perversa la que transforma los cuerpos humanos en objetos al servicio del mercado y economías que, aunque informales, siguen las mismas reglas que la mayoría de los sistemas capitalistas más formalizados.

Las porteadoras son el resultado de políticas migratorias y regulaciones extraterritoriales que visibilizan la objetivación de los sujetos que se produce en la elaboración de leyes. Como describen Feldman y Ticktin, es el régimen de gobierno el que «no sólo regula la intervención en la humanidad como [un] objeto», sino que también «define qué personas 'cuentan' como sujetos para este objeto» (Feldman y Ticktin, 20Io:I8). Como resultado, aquellos que no entran en la categoría de sujetos de humanidad son inmediatamente apartados como objetos, con todas las implicancias deshumanizantes que esta categoría conlleva.

Ceuta, un limboscape con porosidad selectiva Al considerar las terminales de seguridad israelíes, Weizman (2006:94) afirma que «la doctrina de seguridad (...) levanta muros y conceptualmente los 'desmuraliza', (...) concibe los muros como transparentes y permeables por un lado (pero no por el otro)». En la reja de Ceuta, estos gestos de 'desmuralización' no responden a una lógica de visibilidad para algunos e invisibilidad para otros, sino a una porosidad selectiva que crea un comportamiento diferencial en relación al origen del inmigrante.

Pero si la reja/muro está ahí para proteger algo, ¿qué es lo que estas complejas infraestructuras físicas y legales están protegiendo? Considerando ambas trayectorias, se puede argumentar que la porosidad selectiva refleja la «polisemia institucional de la frontera como una línea de separación» (Balibar, 20II:5). Además, las contradictorias articulaciones espaciales reveladas dentro de esa frontera muestran que el límite se vuelve prácticamente indiscernible desde el espacio (tanto geográfico como institucional) que encierra y protege.

Así, es necesario preguntarse, ¿hasta qué punto la paradójica 'coincidencia' que representa Ceuta es el resultado 
de una construcción legal y política, planificada o no? Más aún, asumiendo que es un resultado inesperado de la mate. rialización de todas esas infraestructuras legales y físicas, ¿cuál es la responsabilidad de esos acuerdos legales sobre la controvertida realidad física de esa tierra, esas trayectorias y esos cuerpos? ARQ

\section{Notas / Notes}

I La disputa sobre la soberanía de Gibraltar se basa principalmente en la territorialidad de las aguas y la demanda sobre el territorio continúa hasta hoy debido a nuevas acusaciones entre ambos países, con la ONU actuando como mediador.

2 Sitio web ESF. Accedido el 29 de noviembre, 2015. www.concertina.es

3 Con una superficie total de $19,48 \mathrm{~km}^{2}$, Ceuta tiene un perímetro de 30 $\mathrm{km}$, de los cuales 8 constituyen su frontera terrestre con Marruecos (Ferrer-Gallardo y Espiñeira, 2015:25I).

4 El término "apátrida" al menos reconocía el hecho de que estas personas habían perdido la protección de su gobierno y exigía acuerdos internacionales para salvaguardar su estatus legal. El término posguerra "personas desplazadas" se inventó durante la guerra con el propósito expreso de liquidar la apatridia de una vez por todas al ignorar su existencia. El no reconocimiento de la apatridia siempre significaría la repatriación, es decir, la deportación a un país de origen. Ver: Arendt, 1966:279.

5 Como se estipula en el artículo I44.b de la Constitución Española las ciudades de Ceuta y Melilla, sin dejar de ser Entidades Locales, disponen de sendos Estatutos de Autonomía aprobados, respectivamente, por las Leyes Orgánicas I/I995 y 2/I995, de I3 de marzo. www.agenciatributaria.es

\section{Bibliografía / Bibliography}

Ag A в в N, Giorgio. Means Without End: Notes on Politics. Minneapolis: University of Minnesota Press, 2000.

ARENDT, Hannah. «The Decline of the Nation State and the End of the Rights of Man,» in The Origins of Totalitarianism. San Diego: Harcourt Brace \& Company, I966.

BALFOUR, lan and CADAVA, Eduardo. «The Claims of Human Rights: An Introduction,» South Atlantic Quarterly 103, no. 2/3 (Spring/ Summer 2004).

BALı BAR, Etienne. «Strangers as enemies. Walls all over the World, and How to Tear them Down,» conferencia en Columbia University, 3 de noviembre de $201 \mathrm{I}$.

FELDMAN, Ilana and TICKTIN, Miriam. «Introduction» in Feldman and Ticktin eds. In the Name of Humanity: The Government of Threat and Care. Durham and London: Duke University Press, 2010.
FERRER GALLARDO, Xavier. «Acrobacias fronterizas en Ceuta y Melilla. Explorando la gestión de los perímetros terrestres de la Unión Europea en el continente africano,» Doc. Anàl. Geogr. 5I, 2008.

ferrer Gallardo, Xavier and espiñeira, Keina R. «Immobilized between Two EU Thresholds: Suspended Trajectories of Sub-Saharan Migrants in the Limboscape of Ceuta,» in Martin Van Der Velde and Ton Van Naerssen eds. Mobility and Migration Choices. Thresholds to Crossing Borders. Surrey, England; Burlington, vт: Ashgate Publishing, 2015. WEIZman, Eyal. «Seeing through Walls: The Split Sovereign and the One-Way Mirror», Grey Room 24 (Summer 2006).

\section{Maite Borjabad López-Pastor}

<mborjabad3935@columbia.edu>

Arquitecto, Eт SA M, Universidad Politécnica de Madrid. Realizó estudios en el Illinois Institute of Technology, donde fue galardonada con el Dean's List of Excellence. M.Sc Critical, Curatorial and Conceptual Practices in Architecture, GSA P P, Columbia University, Nueva York. Ha trabajado en The Metropolitan Museum y la Arthur Ross Architecture Gallery (NY). Como curadora independiente, ha desarrollado diversas exposiciones, simposios, happenings y eventos centrados en distintos formatos de prácticas espaciales críticas en colaboración con la Fundación Emily Harvey, el New Museum Incubator (NY), Tabakalera (San Sebastián) o La Casa Encendida (Madrid), entre otros. Su trabajo ha sido publicado en diversos medios como Pin-Up Magazine, Domus, Dezeen, Metalocus, Yorokobu, e-flux, el Chicago Tribune o El Cultural. 